\section{Medication Therapy Management Programs: To Optimize Pharmacy Outcomes}

\section{To the Editor:}

The Medicare Prescription Drug, Improvement, and Modernization Act of 2003 (MMA) has introduced Medication Therapy Management (MTM) as a program requirement for prescription drug plan sponsors and Medicare Advantage plans under the new prescription drug benefit that will become available in 2006. MTM is defined as a program of drug therapy management and medication administration. The goal of MTM is to ensure that drugs provided to eligible beneficiaries are appropriately used to (a) optimize therapeutic outcomes through improved medication use and (b) reduce the risk of adverse events, including adverse drug interactions.

The potential benefits of MTM appear to be clear. There are numerous programs and initiatives throughout the country in a wide range of settings that fall within the continuum of activities and services that would be defined as MTM services. Nevertheless, MTM is in its formative stage with no specific "best practices" or quality assurance standards yet articulated or fully evaluated.

The American Society of Health-System Pharmacists (ASHP) and the Academy of Managed Care Pharmacy (AMCP) convened two Executive Sessions on Medication Therapy Management programs to bring together stakeholders with both an understanding of the concepts of medication therapy management and the need to operationalize those concepts. During these sessions, many aspects of MTM were discussed. Active dialogue was encouraged as participants were asked to share opinions on opportunities and issues related to the implementation of MTM under MMA. Consensus was not sought on any aspect of the discussion. The following document is a summary of the discussion.

Judith A. Cahill, CEBS

Executive Director

Academy of Managed Care Pharmacy

Henri R. Manasse, Jr., PhD, ScD

Executive Vice President and Chief Executive Officer American Society of Health-System Pharmacists

Editor's note: This summary is published simultaneously in the March 15, 2005, issue of the American Journal of Health-System Pharmacists.

\section{Summary of the Executive Sessions on Medication Therapy Management Programs}

Bethesda, Maryland

June 14 and August 18, 2004

Pharmacists and other health care professionals, as well as Medicare beneficiaries, had reason to welcome passage of the
Medicare Prescription Drug, Improvement, and Modernization Act of 2003 (Public Law 108-173). The act requires, among other things, that all prescription drug plan sponsors participating in Medicare Part D and Medicare Advantage plans providing a drug benefit under Part $C$ offer a medication therapy management (MTM) program to help ensure that drugs are used to "optimize therapeutic outcomes through improved medication use and to reduce the risk of adverse events, including adverse drug reactions," in certain high-risk patients.

The Medicare beneficiaries targeted by the MTM program are those who have multiple chronic conditions, are taking multiple medications, and are likely to incur high drug costs. The opportunity for the MTM program to influence the quality and cost of care purchased by Medicare is extraordinary. Just under $80 \%$ of Medicare beneficiaries have multiple chronic conditions, and $20 \%$ of Medicare beneficiaries have five or more chronic conditions, with the latter group accounting for over two thirds of Medicare spending. ${ }^{1}$ In general, health care spending for a person with one chronic condition is 2 times greater than spending for someone without any chronic conditions, while spending is about 14 times greater for someone with five or more chronic conditions. For these patients, the law provides access to a range of professional services designed to improve the safety and efficacy of drug therapy. For pharmacists, this presents a new and important opportunity to collaborate with patients and other health care professionals to improve medicationuse outcomes. The federal government hopes that the act will not only bring about better patient outcomes but also will moderate the rate at which health care and drug costs have increased in recent years.

The Centers for Medicare and Medicaid Services (CMS), which will administer the new benefit, is well aware of the complex issues surrounding the act's implementation. In fact, the proposed rule implementing the 2006 prescription drug benefit, issued by CMS on July 24, 2004, is unusual in that it seems to raise as many questions as it answers. As CMS develops the final regulations governing the act's implementation, it is seeking input on such major issues as the criteria for eligibility for MTM services, the scope of core services to be provided, and the qualifications of service providers.

To provide an opportunity for individuals in the private sector who will be responsible for implementing the MTM programs to explore these issues, the American Society of Health-System Pharmacists (ASHP) and the Academy of Managed Care Pharmacy (AMCP) recently convened two Executive Sessions on Medication Therapy Management Programs. The first session was held on June 14, 2004; the second took place on August 18. Both sessions were held at ASHP headquarters in Bethesda, Maryland. Participating were approximately 25 leaders representing pharmacy benefits management companies (PBMs), health plans, health care organizations, and state and national pharmacy organizations. 
Participants engaged in frank and productive discussions but remained fully cognizant of antitrust requirements.

This report summarizes for the pharmacy community and drug plan sponsors the group's discussions with the aim of facilitating the successful implementation of MTM programs. Any specific views expressed were those of the individuals expressing them, and no endorsement is implied by other participants or the organizations that employ them.

\section{First Executive Session}

In welcoming remarks on June 14, ASHP Executive Vice President and Chief Executive Officer Henri R. Manasse, Jr. noted that an executive session is a forum designed to provide a safe space for the confidential discussion of critical social issues. The topic central to MTM-the safe and effective use of prescription medications by a vulnerable segment of the Medicare population-is one such issue. The participants in the executive session are leaders who are capable of using their spheres of influence to disseminate and advocate for any recommendations that emerge from the day's discussions. Although ASHP and AMCP would publish the meeting proceedings in their respective journals, none of the comments would have attribution, and the report summarizing the proceedings would be submitted to all participating organizations for review before publication.

One purpose of the first session was to provide an introduction to the scope and variety of current MTM-type services and the settings in which they are offered. Representatives from an academic health center, a state Medicaid pharmaceutical case management program, a health maintenance organization, and three PBMs shared information about their programs that are relevant to MTM. Participants then discussed elements that are key to the successful participation of drug plan sponsors in an MTM program, namely the core elements of an MTM program, enrollee eligibility criteria, and the similarities and differences between the services provided by integrated networks and those offered by stand-alone drug plan sponsors.

At the meeting's end, participants agreed that they had engaged in a productive discussion and expressed the desire to reconvene.

\section{Second Executive Session}

As the August 18 meeting opened, AMCP Executive Director Judith A. Cahill summarized some basic assumptions and established objectives for the day. These objectives were as follows:

- Provide an opportunity for interested parties to discuss and gain a better understanding of the MTM program requirement in the Medicare Prescription Drug, Improvement, and Modernization Act and the proposed regulations that would facilitate implementation of that requirement.

- Develop a broad framework for drug plan sponsors to meet the MTM program requirement, with a focus on defining the core elements of an MTM program and measurement criteria to determine whether a plan's MTM program is achieving the objectives as set forth in the statuteoptimization of therapeutic outcomes for beneficiaries by promoting appropriate medication use, detection and reduction of adverse drug events, increased adherence to medication regimens, and identification of patterns of overuse and underuse of medications.

- Encourage meeting participants to consider the discussions that occurred during these meetings as their organizations develop comments on the MTM program requirement for submission to CMS on the proposed regulations and as they begin to develop their organizations' MTM program.

Cahill stated that the general design of the MTM program defined in the proposed rule shows that CMS had heeded the advice offered by the Pharmacist Provider Coalition (PPC), a group of pharmacy organizations whose goal is to ensure that pharmacists are recognized as providers in Medicare Part B. PPC had endorsed and shared with CMS seven core principles that it believes should guide CMS and drug plan sponsors in the implementation of the MTM provisions of the Part D prescription drug benefit.

In the preamble to the proposed rule, CMS has shown that it is flexible and open to suggestions. It has not produced a dictum or a directive; indeed, noting lack of extensive experience in the design of MTM programs and wanting to encourage innovation, CMS chose to suggest options and welcome comments on how the programs can be more sharply defined.

Although much is uncertain, the preamble makes several important points that served as a foundation for the day's discussions. With respect to defining core MTM program elements, the preamble mentions several services in addition to those named in the statutory language, such as assessment of health status, education of family members, coordination of therapy, and collaboration among providers. The preamble reiterates that MTM services are distinct from drug dispensing. It also underscores that MTM services must be patient-specific.

The statute sets forth three eligibility criteria: Enrollees must (1) have multiple chronic diseases, (2) take multiple Part Dcovered drugs, and (3) be likely to incur annual drug costs that exceed a certain level. CMS proposes to give drug plan sponsors the discretion to further define the beneficiary categories as part of their benefit design, even for the criteria the statutory language explicitly designates to be defined by the U.S. Secretary of Health and Human Services.

The law designates pharmacists as MTM providers, but it also allows others to provide MTM services. It does not define who these other providers might be.

The law provides that drug plan sponsors shall pay MTM providers on the basis of the "time and resources used" to provide services, but the proposed rule does not give drug plan sponsors any additional guidance. 
MTM programs are to be included as components of the Medicare drug benefit that would be offered by prescription drug plan sponsors under Part D and Medicare Advantage plans providing a drug benefit under Part C; there is no separate payment for the MTM program. This poses a particular challenge for drug plan sponsors that offer a drug-only benefit because they will not reap the benefit MTM promises in terms of reduced visits to physicians' offices and hospital emergency rooms. In fact, the program may at times increase drug costs; therefore, some participants recommended that CMS consider allocating a separate payment for MTM services, even though that is not consistent with the statutory requirement.

CMS is requesting information on current best practices, quality-assurance considerations, and the development of tools, such as scorecards, that will enable beneficiaries to compare MTM programs offered by drug plan sponsors.

\section{Discussion Themes}

On the basis of comments made at both sessions, it was evident that participants held a number of baseline assumptions. Among the most important are the following:

- With MTM, the government has made a major commitment to the safety of drug therapy. The program acknowledges a societal imperative to take measures to effect optimal patient outcomes for a group of high-risk individuals. As it enters this new territory, CMS can benefit from guidance from the pharmacy profession and drug plan sponsors. Assistance in defining core MTM services is essential. Meeting participants agreed that MTM should go beyond traditional pharmacy services, dispensing and counseling, and brown-bag medication reviews, though recognizing the value of such services.

- MTM programs offer an enormous opportunity to pharmacy, and pharmacy is, in general, prepared for the challenge. The basic structure or capacity for realizing MTM goals is in place; however, it will have to be intensified. Working relationships must be modified and expanded, and contractual relations must be established. The profession may also have to consider creating new credentialing programs and enriching the pharmacy curriculum, particularly in such areas common to the Medicare population (e.g., geriatrics and services for disabled patients).

- Although some MTM services will reduce drug costs, MTM programs cannot always be expected to do so; some compliance and adherence programs designed to optimize therapeutic outcomes may result in higher drug spending. CMS and drug plan sponsors cannot expect a quick financial return on investment when drug cost is the only consideration. For this reason, as well as the uncertainties currently surrounding the program, some plans may initially be reluctant to apply to participate.

- Because the investment is large and the stakes are high,

\section{Medication Therapy Management Services Definition} (as defined by 11 national pharmacy organizations in July 2004)

a. Performing or obtaining necessary assessments of the patient's health status

b. Formulating a medication treatment plan

c. Selecting, initiating, modifying, or administering medication therapy

d. Monitoring and evaluating the patient's response to therapy, including safety and effectiveness

e. Performing a comprehensive medication review to identify, resolve, and prevent medication-related problems, including adverse drug events

f. Documenting the care delivered and communicating essential information to the patient's other primary care providers

g. Providing verbal education and training designed to enhance patient understanding and appropriate use of his or her medications

h. Providing information, support services, and resources designed to enhance patient adherence with his or her therapeutic regimens

i. Coordinating and integrating medication therapy management services within the broader health care management services being provided to the patient

accountability will be essential. Measuring short- and longterm outcomes and ensuring individual and organizational competence will be vital. Pharmacy-management-specific criteria are also essential. The lack of data and data latency will be problematic but not insurmountable.

- CMS's desire for input from pharmacy will encourage the innovative thinking needed to ensure high-quality, costeffective MTM services.

\section{Core Elements of MTM Programs}

The purpose of an MTM program, according to the preamble of the proposed regulations, is to "provide services that will optimize therapeutic outcomes for targeted beneficiaries." Such programs may include elements designed to promote enhanced enrollee understanding, increased enrollee adherence, and detection of adverse events. Possible services noted in the preamble include performing patient health status assessments, formulating prescription drug treatment plans, managing highcost and specialty medications, evaluating and monitoring patient response to drug therapy, providing education and training, coordinating medication therapy with other care management services, and participating in state-approved collaborative drug therapy management.

Seven of the services listed in the proposed rule also appear in a document entitled "Medication Therapy Management Services Definition," which was approved by a group of 11 national pharmacy organizations in July 2004. The box (above) lists the services agreed upon by the pharmacy organizations; items appearing in italics (i.e., items a, b, d, f, g, $h$, and i) also appear in the proposed rule. 
Attendees generally agreed that MTM could include a broad scope of services beyond the traditional counseling associated with dispensing services and that pharmacy should be able to not only assume responsibility for providing these services but also be held accountable for the outcomes of such services.

At the same time, members noted that the elements would require fine-tuning to be useful. Such elaboration would be needed both to limit the scope of work and to make it possible to assess outcomes. For example, in its application, a drug plan sponsor might state that it would be responsible for the prevention of medication-related problems for a designated list of medications but not for drugs in general.

A key service not mentioned on either list was prevention. In keeping with their professional mission and the goal of this legislation, pharmacists should, it was suggested, take a more proactive role in preventing the progression of chronic disease. To emphasize pharmacy's expanded role in the continuity of care, the suggestion was made to include in the list references to services that can be provided at specific stages in the care process where gaps commonly occur. One such time is at discharge from a hospital.

While participants agreed that this spectrum of services should be available to every eligible enrollee, each practice setting may not provide all services. For participating drug plan sponsors, the goal will be to design a system in which a patient meeting the plan's criteria will be ensured of receiving appropriate services, whether from the plan itself or through a contract or subcontract between the plan and another entity or individual.

\section{Identification of Target Beneficiaries}

MTM services are of potential value to every patient, but drug plan sponsors are not required to provide them to every beneficiary. Resource allocation is essential. Drug plan sponsors must develop a rationale that establishes the criteria on which they will determine MTM eligibility, with the understanding that, for a variety of reasons, some patients will not receive services from which they would otherwise benefit.

The proposed rules define eligibility broadly, stating that MTM beneficiaries are enrollees who have multiple chronic diseases, are taking multiple Part D-covered drugs, and are likely to incur annual drug costs that exceed a certain level.

Multiple chronic diseases. Existing data on the incidence and prevalence of chronic diseases in the Medicare population should be a prime source that plans can use in defining the covered disease states. Also important is the availability of treatment guidelines and firm evidence on the effectiveness of drug therapy in those treatment protocols. The use of evidencebased medicine and outcome indicators should make it possible to better assess outcomes. For example, for diseases such as congestive heart failure and asthma, there are indicators that clearly show better outcomes associated with certain drugs.
When determining the conditions on which their programs will focus, drug plan sponsors must bear in mind these types of considerations.

Multiple drugs. The extent of multiple drug use among the elderly and the wide differences in the costs of drug products make setting this criterion exceptionally difficult. A patient may be taking only one drug, but, if it is the wrong drug, this could have far-reaching implications for that patient's health outcome. Rather than establishing a cutoff definition of drugs on the basis of numbers alone, it might be beneficial for plans to use additional criteria beyond "the use of multiple medications." These could relate, for example, to particular combinations of drugs, the nonuse of indicated drugs for certain diagnoses, or situations in which patients stop and resume therapy with drugs used to treat chronic diseases.

Costs. Defining eligibility on the basis of a single financial figure is problematic in many respects. The desirable figure for such a determination depends on one's perspective. The prescription drug plan, the government, and taxpayers would want a different figure, for example, than would beneficiaries. Moreover, it is not just the big-ticket items that contribute to overall health care costs. A patient who spends relatively little on drugs can be at risk for high overall health care costs if the medications are not used appropriately.

If a cost threshold is set, a drug plan sponsor would probably not want to design its plan so that an enrollee becomes eligible for MTM only after having reached that set dollar amount. It would be preferable to use modeling to determine that the enrollee was on track to meet or exceed the amount and then initiate MTM to prevent that cap from being reached.

Other criteria. Patient populations other than those meeting the statutory definition of high risk, having multiple chronic diseases, taking multiple medications, and incurring high drug costs might benefit greatly from MTM. Among these are beneficiaries who see multiple physicians and multiple pharmacists or those who otherwise show a lack of evidence of coordination of care. Also, Medicaid-eligible Medicare beneficiaries may pose different socioeconomic or lifestyle-related risks.

Participants noted that drug plan sponsors might want to use predictive modeling tools to help identify targeted patients.

\section{Importance of Outreach}

Identifying potential eligible beneficiaries is only the first step in delivering MTM services. Once these individuals are identified, there must be a structured means to help them access services. This must be multifaceted, using not only the plans to identify beneficiaries but also physician and pharmacist referral.

Beneficiary participation in MTM programs will be voluntary; thus, an individual who meets the enrollment criteria may be identified and informed of the service but refuse to participate. Some informed seniors, on the other hand, might ask their pharmacists whether they can participate. That situation will 
most likely not be the norm at the onset of benefit implementation. It will be necessary to reach out to potential recipients, invite them to participate, and then follow up to ensure service coordination.

As pharmacists reach out to patients, they may confront a stark reality. With respect to pharmacy services, many patients have low expectations. In many cases, their only wish is to be assured that they have received the right medication. MTM gives pharmacists broader responsibilities and will require a corresponding rise in patient expectations.

Perhaps because of their greater dependence on medications, older Americans are often more likely than younger persons to have established a trusting relationship with a pharmacist. Nonetheless, many patients view their physician as the ultimate authority on medication use. Teaching a patient to expect these services from his or her pharmacist will require an educational outreach to beneficiaries and relationship building among pharmacists, physicians, and others in the beneficiary's network of care. The drug plan sponsor must reach out to patients and other caregivers with information about the program and what it can accomplish.

Outreach efforts must touch physicians as well. Physician buy-in is essential to the success of MTM programs. When issues relating to collaborative patient management arise, many physicians say they understand the need for such relationships but note that they are not familiar with the pharmacists practicing in community settings. In such a situation, physicians may be reluctant to collaborate with pharmacists.

Building relationships with physicians is essential. Community pharmacy residency programs may be one small way to build interprofessional trust in the community setting. Other methods must be explored, and MTM, by emphasizing continuity of care, can spur their development.

Finally, outreach efforts should include patients' family members and other nonprofessional caregivers. These individuals are especially important in the provision of care to the elderly population. Caregivers themselves, as well as enrollees, may need MTM services.

\section{A Strengths-Based Approach}

MTM services will be patient-specific, focused on achieving the desired outcomes in an individual patient. Participating drug plans must design their programs and configure their services in the way that will best ensure attainment of this goal. In some cases, the dispensing pharmacist may be the pharmacist who provides all or most of a patient's MTM services; in others, it will not. A patient who purchases drugs through the mail, for example, could receive MTM services from pharmacists on staff with the mail-order service or from a local pharmacy provider. A patient who purchases drugs at a local pharmacy could receive MTM services from another MTM provider.

Meeting participants emphasized the need for a strengths- based approach; drug plan sponsors must develop a plan that capitalizes on its strengths, defines its services, and then lets the patient choose the plan in which he or she wishes to enroll.

Plan sponsors are likely to base their program on existing strengths and capabilities and then make arrangements to ensure that its enrollees can secure needed additional services elsewhere. The plan sponsor would design an MTM program that would identify potential enrollees at the time they entered the plan, bring them into the MTM program, create the scope of services around patients' needs, and use triage, where necessary, to ensure that patients receive necessary services. Some meeting participants noted that a drug plan sponsor with a mail-service (or mail-order) pharmacy may use its clinical pharmacy call center as a starting point for the provision of MTM services. A community pharmacy, or any other setting, would use a similar strengths-based strategy-devising an MTM patient care approach (from identification and enrollment to actual provision of services) based on its current strengths.

There may be service bifurcation as plans call on their different internal strengths and rely on networks or contracts to ensure that enrollees receive specialized services or services that entail the collaboration needed to offer continuity of care. MTM programs will continue to evolve to best meet the needs of individual patients.

\section{Referral for MTM Services}

A comprehensive menu of services is meaningless if it is underused. For this reason, the manner in which services are triggered is a major concern. In preparing its proposal, the drug plan sponsor must articulate its criteria for determining that a patient needs MTM services and provide opportunities for multiple points of referral. Ideally, anyone in the continuum of care should be able to make a referral, including community pharmacists, who are often in an ideal position to identify patients in need of such services.

Reliance on any single source would be too limiting. Experience with other federally supported programs has shown that if referral is dependent on physicians alone, for example, it is likely that many patients will be missed and that MTM services will be underused.

These points of referral might be episodic and related to a particular point in the continuum of care. For example, they might occur when a patient is discharged from the hospital to home or to an assisted-living facility.

Plans must also develop a plan for determining how they will ensure the delivery of services. The patient should be referred to the provider; the drug plan sponsor would then decide whether its model accommodates the service requests.

To ensure continuity of care, it was noted that recommendations made through MTM programs would need to be coordinated with the initiatives of the new Chronic Care Improvement Program. 


\section{Qualifications of MTM Providers}

The act does not set educational or experiential criteria for pharmacists or other qualified providers of MTM services. Pharmacists seem to be seen as the primary providers of MTM services; however, this is not explicit. Meeting participants agreed that drug plan sponsors would need some flexibility to determine who qualifies under their program and might con-sider prospective providers' training, education, and experience.

A graduate of a doctor of pharmacy (PharmD) program should be capable of providing core MTM services, most group members agreed, even though a recent graduate may lack the in-depth experience needed to handle the most complex cases. Some new graduates and seasoned practitioners may need additional education and training in geriatrics to meet the needs of this population. Pharmacists with certification in certain specialties are well prepared to provide core MTM services in those specialty areas, although their numbers are insufficient to limit the provision of MTM services to these pharmacists. Pharmacists with a bachelor of science degree and no certification, but with years of experience in the appropriate setting, are also well qualified to provide MTM services.

Meeting participants suggested several ways of tackling these interlocking challenges. First, any drug plan sponsor should ensure that its work force is diverse and complementary in terms of experience, skills, and knowledge. Plans can set an educational and experiential profile and use it as a reference point when hiring new employees or contracting with communitybased pharmacists or other MTM providers. Plans should offer incentives that encourage their work force to attain new levels of achievement.

It was suggested that the profession may need to explore new credentialing paradigms. The entry-level PharmD degree and specialty certification have brought a new level of excellence to the profession. Other forms of recognition, tailored to the needs of MTM programs, could be developed.

One participant suggested that CMS could eventually develop conditions of participation for plans. Given the diversity of program settings and patients' needs, defining meaningful baseline criteria would be difficult.

Ensuring that pharmacists are prepared to provide highquality MTM services in sufficient capacity to meet the needs of MTM programs is ultimately a deployment issue for the entire profession. It will require collaboration among employers and educational institutions. The latter must make sure that their curricula prepare graduates adequately to provide MTM services. Employers must realize that pharmacists, no matter how knowledgeable and skilled, must practice in a professional work environment that is conducive to providing high-quality patient care services in order for MTM to be successful.

The proposed rule states that, in addition to pharmacists, other health care providers can have roles in MTM. Who these professionals might be and what roles they might assume will depend on enrollee characteristics, core elements, and health care settings defined elsewhere in the drug plan sponsor's proposal.

\section{Ensuring Accountability}

Those attending the executive sessions had no doubt that CMS will hold drug plan sponsors and those who work for or contract with them accountable for the outcomes of MTM services. They prefaced their discussions of accountability with two overarching thoughts. The first is that being held accountable for the outcomes of MTM services is a landmark-pharmacists will move beyond simply being responsible for such services. The second idea, expressed succinctly by one attendee, is that good management requires accountability mechanisms: "You cannot manage what you cannot measure."

Although agreeing on the need for accountability, participants had many questions concerning how it will be ensured under MTM. A basic question is: Who will be held accountable? The plan and the provider may not be the same entity. If the drug plan sponsor is held accountable, how will it delegate accountability to the provider?

Outcomes measures for an integrated network will differ from those of a freestanding plan. Although some measures will be shared, others will not. Issues relating to collaboration and continuity of care, for example, must be addressed by integrated plans. Sponsors of such plans are also interested in total outcomes, expressed by such criteria as reductions in overall hospitalizations. Stand-alone plans do not currently have the medical data on which to evaluate the role their services play in achieving such a goal.

One solution, stimulated by MTM discussions, is to design plans in a manner that will blend the population-based focus of PBMs with the traditional individual responsibility of the provider. MTM will require the formation of networks. To make the program work, a means must be found to merge pharmacy claims data with medical data.

The need for data is an overriding problem. National data, available from the National Committee for Quality Assurance's HEDIS (Health Employee Data and Information Set) and Medicare's quality improvement organizations, can be tapped. High-quality outcomes measures, such as the Study of Clinically Relevant Indicators for Pharmacological Therapy (SCRIPT) measures developed by CMS and the Medication Appropriateness Index developed by the Veterans Affairs Measurement Excellence and Training Resource Information Center, will likewise be helpful, particularly when geared specifically to the geriatric population.

The bottom line with respect to data, as one participant noted, is that "you've got to work with what you have." Good work can be done in ensuring accountability, even though the data are not perfect or complete. At the same time, given the 
importance of data sharing, information technology will be crucial to the success of programs from multiple standpointsfor transferring information among health care providers, between plans and providers, and between providers and patients and for capturing claims data and other information needed for measuring outcomes and determining costs. CMS may want to consider investing in a means to ensure efficient data transfer that can be used by all program participants. Such a tool would be invaluable in ensuring interprogram consistency.

\section{Need for MTM-Specific Outcome Criteria}

Ultimately, CMS will most likely be the source of the data most needed by drug plan sponsors to assess both long- and shortterm outcomes of MTM programs. If, as these programs get under way, CMS could develop criteria to compare the impact of different MTM programs on Medicare beneficiaries' overall health needs, it would create a more complete picture with which to measure MTM programs' success. CMS is developing the capacity to issue monthly claims feeds. It will provide these data to the Chronic Care Improvement Program participants and could also make them available to drug plan sponsors.

In light of the absence of direction from CMS and access to historical data, the profession itself should develop a set of measures that drug plan sponsors and CMS can use to assess the success of MTM programs. Participants from pharmacy organizations suggested that they could, through their respective foundations, create a subcommittee that could survey the literature, hold a research-based discussion, and develop such indicators.

The assumption underlying any such effort should be that a reduction in overall drug costs is just one criterion on which to rate the success of a program. Some aspects of MTM will increase drug costs.

Working on this premise, participants sought to identify some cost-, condition-, and process-specific guidelines that could be used as criteria to assess MTM services. Among those named were adherence to the medication regimen and decreases in rehospitalization rates. Some criteria can be conditionspecific; however, given that enrollees will have multiple conditions and that the effects of these conditions are more than additive, focusing on conditions alone yields limited data. The National Center for Health Statistics has data on Medicare beneficiaries that may be helpful in setting these types of criteria.

Patient satisfaction is an outcome measure. Plans will need to devise ways to secure patient-satisfaction data from enrollees themselves. If patients are not satisfied, they will change plans. Such measurement tools need not be overly sophisticated. Questions such as "Was your pharmacist responsive to your needs?" or "Did you have access to the pharmacist?" could serve as a starting point. Plans should likewise survey physicians to gauge their satisfaction with pharmacy services.

As valuable as discrete indicators are, there is a danger that, in focusing on them, the overall objective of enhanced patient care through improved medication use could be lost. Plans cannot focus too much on certain indicators, no matter how informative, at the expense of others. Ensuring an appropriate balance should be the responsibility of drug plan sponsors.

\section{Economic Incentives for Participation}

Among the many factors that surround the design and implementation of MTM services, none is more important—or less predictable-than how many drug plan sponsors will decide to apply to participate in the programs, particularly at the outset.

Because MTM programs are currently so loosely defined, many uncertainties exist. Noting that the program may add financial risk, one participant suggested that participating plans might want to insure the MTM segment of their plans against financial loss. Yet another issue is the fundamental difference in care needs, even among this identified high-risk population. One participant praised the program structure because, by offering the possibility of risk adjustment, it will offer plans an incentive to manage complex patients. Previously, plans might have been reluctant to accept such patients because of a fear of adverse economic consequences. Under MTM, a plan that successfully manages patients with diabetes, for example, may choose to try to attract this patient group because of the risk adjustment.

Some stand-alone plans may see few economic incentivesand a great deal of work-associated with offering an MTM program. Others may view the program as a way to create win-win situations for themselves and their beneficiaries.

Participants presumed that CMS will expect a return on its investment, although it has also made a commitment, with MTM, to safe drug use. The Bush administration has placed a strong emphasis on competition in the marketplace, and CMS may see program applications as an opportunity to identify and test a broad range of programs and ensure that it gets the best outcomes for the smallest investment. For the drug plan sponsor, the goal will be to submit a bid that is low enough to get the business but high enough to make participation feasible.

With respect to economic incentives, participants underscored two important elements. First, CMS must assess the MTM component of proposals separately from drug costs and must use consistent, well-defined criteria in making these assessments. The MTM segment of a plan's proposal should account for a specific percentage of the overall score. If MTM services do not receive separate treatment, participants will lack the incentive to perform them well.

Second, CMS must set realistic expectations. If a plan proposes to serve 3\% of its Medicare enrollees, CMS cannot demand that it serve $6 \%$, unless CMS is willing to accept a corresponding decrease in the breadth of services provided to each of these individuals as well as less-desirable results. Tradeoffs will have consequences. 


\section{Moving Forward}

Asked what solid take-home points they had gained from the meeting, meeting participants stated that MTM was a "valuable" program and an opportunity to "convince the world that pharmacy has something to offer." They urged that the profession take advantage of the opportunity CMS has made available to help define the criteria under which the program will operate. "We can't leave it to chance," one participant cautioned. Many applauded the program for its potential to improve care coordination for a group of enrollees among whom fragmentation of services is a long-standing problem.

Uncertainties abound-it is a "system waiting to be defined." Through collaborative action, an open-minded approach, and commitment from CMS and the profession of pharmacy, the needed definitions will gradually emerge. The stakes are high. "How we adjust," noted one participant, "will be a measure of our profession."

Finally, participants concurred that MTM programs mark an evolution, rather than a revolution, in pharmacy services. The basic components of MTM are in place. Successfully implementing MTM will challenge programs to become familiar with the legislation, assess their strengths, and build on those strengths to broaden pharmacy's role in ensuring safe drug use, the cost-effective use of resources, and optimum health outcomes.

\section{REFERENCE}

1. Partnership for Solutions. Chronic conditions: making the case for ongoing care. Available at: www.partnershipforsolutions.org/DMS/files/chronicbook2004.pdf. Accessed November 5, 2004.

\section{ORGANIZATIONS REPRESENTED AT THE SESSIONS}

- Academy of Managed Care Pharmacy

- Aetna Inc.

- American Association of Colleges of Pharmacy

- American College of Clinical Pharmacy

- American Health Quality Association

- American Pharmacists Association

- American Society of Consultant Pharmacists

- American Society of Health-System Pharmacists

- Anthem Prescription Management, LLC

- Caremark Inc.

- Catalyst Rx

- Express Scripts, Inc.

- Harborview Medical Center

- Humana Inc.

- Iowa Pharmacy Association

- Medco Health Solutions, Inc.

- MedImpact Healthcare Systems Inc.

- Prescription Solutions

- Prime Therapeutics

- UnitedHealth Group (August 18th session only)

- Walgreens Health Initiatives

- WellPoint Pharmacy Management

\section{Quality Articles Require Peer Reviewers- as Well as Authors}

\section{To the Editor:}

In reviewing a recent article for JMCP publication consideration, I had the opportunity to truly understand the importance of peer review. A good general concept is not sufficient-the information must be accurate and presented in a clear manner.

When I was a manager of clinical pharmacy services for a moderate-sized Medicaid managed care plan, there were numerous subjects that I considered to be of critical interest for managed care pharmacists. Volumes of information are published on new medications, and I had limited time to read them. When I did read, I expected the literature to be clear and of high quality. As a result, I selected a limited group of journals that I viewed as my weekly or monthly "must reads." JMCP was one of them.

Until taking the time and seizing the opportunity both to author and participate in peer review for JMCP, I never valued the magnitude of importance that quality peer review provides. As authors, we often become so passionate about our ideas and publication submissions that we do not recognize or appreciate the need for a second opinion or clarification. Peer review provides that opportunity. For instance, in my review of a recent manuscript on the economic impact of the use of various psychotropics, I felt that including critical information related to the rationale behind comparing selected agents would clarify the authors' objective in writing the article. This is one of several suggestions that were made by reviewers with the intent of increasing the clarity of the article.

Managed care pharmacy encompasses an ever-expanding magnitude of clinical areas, and the range of disease states and topics that formulary managers and $P \& T$ committees encounter on a daily basis makes it critical that the articles they read are both comprehensible and accurate. Managed care practitioners rely heavily on the experts in their fields to paint a clear picture of both the concept and the details. For that reason, it is essential that publications such as JMCP have, and continue to nurture, a team approach in the writing and review of high-quality literature. Good authors and focused reviewers working together provide the best chance for production of quality publications. 